\title{
Invasive fungal infection in allogeneic hematopoietic stem cell transplant recipients: single center experiences of 12 years
}

\author{
Ji-min $\mathrm{SHI}^{\S}$, Xu-ying $\mathrm{PEI}^{\S}$, Yi LUO, Ya-min TAN, Ru-xiu TIE, Jing-song HE, \\ Wei-yan ZHENG, Jie ZHANG, Zhen CAI, Mao-fang LIN, He HUANG ${ }^{\text {t\$ }}$ \\ (Bone Marrow Transplantation Center, the First Affiliated Hospital, School of Medicine, Zhejiang University, Hangzhou 310003, China) \\ ${ }^{\dagger}$ E-mail: huanghe@zju.edu.cn \\ Received Jan. 4, 2015; Revision accepted June 9, 2015; Crosschecked Aug. 17, 2015
}

\begin{abstract}
Invasive fungal infection (IFI) is a growing cause of morbidity and mortality among patients after allogeneic hematopoietic stem cell transplantation (allo-HSCT). We retrospectively reviewed the records of 408 patients undergoing allo-HSCTs during the period November 1998 to December 2009, analyzed the incidence and risk factors of IFI, and examined the impact of IFI on overall survival. A total of $92(22.5 \%)$ episodes suffered proven or probable IFI (4 patients were proven, 88 patients were probable). Candida was the most common pathogen for early IFI, and mold was the most frequent causative organism for late IFI. A prior history of IFI, human leukocyte antigen (HLA) mismatch, long-time neutropenia, and acute graft-versus-host-disease (GVHD) were risk factors for early IFI. A prior history of IFI, corticosteroid therapy, cytomegalovirus (CMV) disease, and chronic GVHD were risk factors for late IFI. IFI-related mortality was $53.26 \%$. The 12-year overall survival (OS) rate for IFI was significantly lower than that of patients without IFI (41.9\% vs. $63.6 \%, P<0.01)$.
\end{abstract}

Key words: Invasive fungal infection, Allogeneic hematopoietic stem cell transplantation, Incidence, Risk factors doi: 10.1631 jzus.B1500005

Document code: A

CLC number: R55

\section{Introduction}

Allogeneic hematopoietic stem cell transplantation (allo-HSCT) is an effective and sometimes the only curative therapy for patients with high-risk hematologic malignancies. Due to the therapies preventing and treating graft-versus-host-disease (GVHD) and cytopenia, HSCT recipients have a high risk of acquiring invasive fungal infection (IFI) (Jantunen et al., 1997; Bjorklund et al., 2007).

Over the past decades, multiple changes in transplant procedures and antifungal prophylaxis

\footnotetext{
Corresponding author

$\S$ The two authors contributed equally to this work

* Project supported by the National Natural Science Foundation of China (Nos. 81170526 and 81270640)

(D) ORCID: He HUANG, http://orcid.org/0000-0002-2723-1621

(C) Zhejiang University and Springer-Verlag Berlin Heidelberg 2015
}

have affected the epidemiology of IFI (Neofytos et al., 2009; Kontoyiannis et al., 2010). Although fluconazole prophylaxis has reduced the incidence of IFI caused by Candida albicans, it resulted in a subsequent increase in resistant candidiasis, such as Candida glabrata and Candida krusei. Aspergillus has become the most frequent organism and other infections caused by molds, such as Zygomycetes and Fusariums, also have increased since the 1990s (Neofytos et al., 2009).

Previously identified risk factors of IFI include donor type, GVHD, high-dose corticosteroids, increased ferritin levels, and so on (Fukuda et al., 2003; Thursky et al., 2004; Ozyilmaz et al., 2010; Omer et al., 2013). Epidemiology and risk factors of IFI differ due to the changes in antifungal prophylaxis and treatment in different regions. Exploring these differences may improve management strategies and study design for allo-HSCT recipients. 
Here in our study, we retrospectively reviewed 408 consecutive patients who underwent allo-HSCT between 1998 and 2009 in our center, identified the incidence and risk factors of IFI, and calculated the outcomes.

\section{Subjects and methods}

\subsection{Patients and donors}

We collected a total of 408 patients, 251 males and 157 females aged 8-52 years (a median age of 28 years), who underwent allo-HSCT in our center between November 1998 and December 2009. According to the French-American-British (FAB) criteria, $127(31.13 \%)$ cases were acute myeloid leukemia (AML), 120 (29.41\%) were acute lymphocytic leukemia (ALL), 127 (31.13\%) were chronic myeloid leukemia (CML), and 34 (8.33\%) were other hematologic diseases. Among the allogenic HSCT recipients, $195(47.79 \%)$ received bone marrow transplantation (BMT), whereas $213(52.21 \%)$ received peripheral blood stem cells (PBSCs) with a granulocyte colony-stimulating factor (G-CSF) mobilized. Donors were human leukocyte antigen (HLA)-matched ( $n=$ 298), HLA-mismatched ( $n=82)$, or HLA haploidenticalmatched $(n=28)$. Clinical characteristics of the entire cohort are presented in Table 1.

\subsection{Transplant procedure}

A total of 349 (85.54\%) patients received myeloablative conditioning regimens composed of busulfan (BU) plus cyclophosphamide (CY). The 27 (6.62\%) patients who received HLA haploidentical transplants received $\mathrm{BU} / \mathrm{CY} /$ cytosine arabinoside (Ara-C)/1-(2-chloroethyl)-3-(4-methylcyclohexyl)1-nitrosourea (methyl-CCNU)/anti-thymoglobin (ATG). Reduced-intensity conditioning regimens which were predominantly fludarabine-based combinations were used in 32 cases. In addition, 66 (16.18\%) individuals with HLA-mismatched donors received ATG as part of their conditioning regimen.

\subsection{GVHD prophylaxis and therapy}

In both unrelated and sibling transplantation cohorts, patients received the same GVHD prophylaxis regimen consisting of cyclosporine, mycophenolate mofetil, and short-term methotrexate (MTX).
Table 1 Characteristics of 408 allo-HSCT recipients*

\begin{tabular}{|c|c|c|c|c|}
\hline \multirow{2}{*}{ Parameter } & \multirow{2}{*}{ Total } & \multicolumn{3}{|c|}{ IFI } \\
\hline & & Yes & No & $P$ \\
\hline $\begin{array}{l}\text { Recipient age (year), } \\
\text { median (range) }\end{array}$ & $\begin{array}{l}28.96 \\
(8-52)\end{array}$ & $\begin{array}{l}28.91 \\
(9-50)\end{array}$ & $\begin{array}{l}28.97 \\
(8-52)\end{array}$ & 0.45 \\
\hline Gender, male/female & $251 / 157$ & $62 / 30$ & $189 / 127$ & 0.14 \\
\hline Underlying disease & & & & 0.47 \\
\hline ALL & $120(29.41)$ & $37(30.83)$ & $83(69.17)$ & \\
\hline AML & $127(31.13)$ & $26(20.47)$ & $101(79.53)$ & \\
\hline CML & $127(31.13)$ & $21(16.54)$ & $106(83.46)$ & \\
\hline MDS/AML & $21(5.15)$ & $5(23.81)$ & $16(76.19)$ & \\
\hline NHL & $3(0.74)$ & $0(0.00)$ & $3(100.00)$ & \\
\hline MM & $3(0.74)$ & $1(33.33)$ & $2(66.67)$ & \\
\hline PNH & $1(0.25)$ & $0(0.00)$ & $1(100.00)$ & \\
\hline MAL & $6(1.47)$ & $2(33.33)$ & $4(66.67)$ & \\
\hline Donor type & & & & 0.24 \\
\hline Related & $164(40.20)$ & $30(18.29)$ & $134(81.71)$ & \\
\hline Unrelated & $244(59.80)$ & $62(25.41)$ & $182(74.59)$ & \\
\hline Stem cell source & & & & 0.25 \\
\hline Bone marrow & $195(47.79)$ & $39(20.00)$ & $156(80.00)$ & \\
\hline Peripheral blood & $213(52.21)$ & $53(24.88)$ & $160(75.12)$ & \\
\hline HLA & & & & 0.03 \\
\hline Matched & $298(73.04)$ & $58(19.46)$ & $240(80.54)$ & \\
\hline Mismatched & $110(26.96)$ & $34(30.91)$ & $76(69.09)$ & \\
\hline Conditioning regimen & & & & 0.68 \\
\hline Without ATG & $342(83.82)$ & $77(22.51)$ & $265(77.49)$ & \\
\hline With ATG & $66(16.18)$ & $15(22.73)$ & $51(77.27)$ & \\
\hline
\end{tabular}

* Data are expressed as $n(\%)$, except for recipient age (median (range)) and gender (male/female). IFI: invasive fungal infection; ALL: acute lymphocytic leukemia; AML: acute myeloid leukemia; CML: chronic myeloid leukemia; MDS: myelodysplastic syndrome; NHL: non-Hodgkin's lymphoma; MM: multiple myeloma; PNH: paroxysmal nocturnal hemoglobinuria; MAL: mixed acute leukemia; HLA: human leukocyte antigen; ATG: anti-thymocyte immunoglobuline

Acute GVHD was graded from 0 to IV according to established criteria. Patients who survived for $>100 \mathrm{~d}$ were determined as chronic GVHD, which was graded as limited or extensive. Acute GVHD was treated with high-dose methyl-prednisolone (MP), and ATG was used for patients refractory to MP.

\subsection{Supportive care}

All patients were cared for in rooms with highefficiency particulate air filters. Empiric antibacterial agents for fever and cotrimoxazole as pneumocystis prophylaxis were administered. For the prophylaxis of cytomegalovirus (CMV), ganciclovir or foscarnet sodium was used before transplantation and acyclovir combined with immunoglobulin after transplantation. 


\subsection{Definition, prophylaxis, and therapy of IFI}

IFI was defined as proven or probable according to the European Organization for Research and Treatment of Cancer/Mycoses Study Group (EORTC/ MSG) criteria (Ascioglu et al., 2002). Proven fungal infection required histopathologic findings from biopsied tissues or culture of sterile tissue. Probable infection was considered when patients had both clinical criteria and at least one microbiologic criterion (fungus was identified from sputum or bronchoalveolar lavage fluid). Infections were classified according to occurrence timing after transplantation: early IFI ( $\leq 100 \mathrm{~d}$ after transplantation) and late IFI (Group I: 100-180 d; Group II: $>180$ d after transplantation).

From November 1998 to December 2007, fluconazole $(400 \mathrm{mg} / \mathrm{d}$, oral (p.o.)) was given to all HSCT recipients from Day -7 to Day +90 post-HSCT, with dosages adjusted on the basis of their renal function. After January 2008, patients with a history of IFI pre-HSCT received itraconazole $(200 \mathrm{mg} / \mathrm{d}$, intravenous (i.v.)) during neutropenia $\left(<1.0 \times 10^{9} \mathrm{~L}^{-1}\right)$, and were changed to oral fluconazole or itraconazole after neutrophil recovery $\left(>1.0 \times 10^{9} \mathrm{~L}^{-1}\right)$. Patients with persistent fever refractory to broad-spectrum antibiotic treatment were also provided with antifungal therapy, usually itraconazole, caspofungin, voriconazole, or amphotericin B.

\subsection{Statistical methods}

Continuous variables were compared between groups using Student's $t$ test or Wilcoxon rank sum test. The Chi-square test or Fisher's test was used for categorical variables. Univariate analysis and multivariate analysis were performed, and Cox proportional hazard models were estimated to assess risk factors for IFI and survival rate. Kaplan-Meier estimates were computed for survival, and a stratified log-rank test was used to compare these groups. $P<0.05$ was considered significant. All analyses were conducted using SAS 9.2 and ATATA 11.0.

\section{Results}

\subsection{Clinical outcomes after allo-HSCT}

Clinical outcomes after allo-HSCT among 408 patients were analyzed and summarized in Table 2 . The median follow-up period was 28 (range 1-145) months. All patients achieved hematopoietic recon- stitution, with neutrophil engraftment $\left(>0.5 \times 10^{9} \mathrm{~L}^{-1}\right)$ on Day +14 (range $+7-+31 \mathrm{~d}$ ) and platelet engraftment $\left(>20 \times 10^{9} \mathrm{~L}^{-1}\right)$ on Day +18 (range $\left.+8-+144 \mathrm{~d}\right)$. The cumulative incidence rates of acute GVHD Grades I to II and Grades III to IV were $35.5 \%$ and $8.1 \%$, respectively. The cumulative incidence of chronic GVHD was 39.2\% (29.9\% limited and 9.3\% extensive). Positive CMV antigenemia was observed in $35.5 \%$ of the recipients. Thirty-nine patients developed CMV disease, including pneumonia, enteritis, and cystitis.

Table 2 Clinical outcomes after allo-HSCT ${ }^{*}$

\begin{tabular}{lcccc}
\hline \multirow{2}{*}{ Parameter } & Total & \multicolumn{3}{c}{ IFI } \\
\cline { 3 - 5 } & & Yes & No & $P$ \\
\hline Day for engraftment & & & & \\
ANC $>0.5 \times 10^{9} \mathrm{~L}^{-1}$ & $14(7-31)$ & & & \\
PLT $>20 \times 10^{9} \mathrm{~L}^{-1}$ & $18(8-144)$ & & & $<0.001$ \\
aGVHD & & & & \\
Grades I-II & $145(35.54)$ & $49(33.79)$ & $96(66.21)$ & \\
Grades III-IV & $33(8.09)$ & $12(36.36)$ & $21(63.64)$ & \\
cGVHD & & & & $<0.001$ \\
Limited & $122(29.90)$ & $32(26.23)$ & $90(73.77)$ & \\
Extensive & $38(9.31)$ & $22(57.89)$ & $16(42.11)$ & \\
CMV & & & & $<0.001$ \\
CMV viremia & $144(35.29)$ & $42(29.17)$ & $102(70.83)$ & \\
CMV disease & $39(9.56)$ & $17(43.59)$ & $22(56.41)$ & \\
\hline
\end{tabular}

${ }^{*}$ Data are expressed as $n(\%)$, except for day for engraftment (median (range)). IFI: invasive fungal infection; ANC: absolute neutrophil count; PLT: platelet; GVHD: graft-versus-host-disease; aGVHD: acute GVHD; cGVHD: chronic GVHD; CMV: cytomegalovirus

\subsection{Incidence and clinical features of IFI}

We detected 92 (22.5\%) episodes of IFI in patients after allo-HSCT, with 4 proven cases and 88 probable IFI cases. The median age of these patients was 29 (range 9-50) years. Among the 92 cases, 69\% were males. The cumulative incidence rates of IFI for $100 \mathrm{~d}, 6$ months, and 1 year were $7.87 \%, 5.77 \%$, and $12.60 \%$, respectively (Fig. 1). Candidiasis was responsible for 50 proven and probable episodes $(54.35 \%)$, and mold infection for 42 episodes (45.65\%). Non-albicans caused 52\% (26/50) of candidiasis. Among mold infection, Aspergillus was associated with $76.19 \%$ (32/42) cases (Fig. 2). The median time after allo-HSCT to onset of IFI was 140 (range 30-598) d for invasive candidiasis infection (ICI) and 243 (range 12-2461) d for invasive mold infection (IMI). There was a trend for ICI to occur earlier post-HSCT than IMI. The lung was the most frequently affected site $(69.6 \%, 64 / 92)$, followed by 
oropharynx $(14.1 \%, 13 / 92)$, gastrointestinal tract $(9.8 \%, 9 / 92)$ and urinary tract $(10.9 \%, 10 / 92)$, blood $(3.3 \%, 3 / 92)$, and skin $(1 \%, 1 / 92)$, and eight cases had more than one site affected.

According to the timing after allo-HSCT, 27 $(29.35 \%)$ cases were diagnosed as early IFI $(\leq 100 \mathrm{~d})$ and $65(70.65 \%)$ cases were diagnosed as late IFI ( $>100 \mathrm{~d})$, of which 43 (66.15\%) developed later than $180 \mathrm{~d}$ after transplantation. Candida accounted for $66.67 \%(18 / 27)$ and $49.23 \%$ (32/65) of early and late IFIs, respectively. However, this difference did not reach statistical significance. We further grouped late IFI to Group I $(<+180 \mathrm{~d})$ and Group II $(>+180 \mathrm{~d})$, revealing that the probability of Candida among Group I was significantly higher than that among Group II (72.72\% vs. 37.21\%; Fig. 2).

\subsection{Risk factors for IFI}

Risk factors by univariate analysis for the development of IFI during each time period are presented in Table 3. HLA mismatch, sustained neutropenia $\left(<0.5 \times 10^{9} \mathrm{~L}^{-1}\right)$ lasting $>2$ weeks, CMV infection, and severe acute GVHD were significant risk factors for early IFI. Chronic GVHD and CMV disease increased risk for late IFI. A prior history of IFI and corticosteroid therapy associated with increased risk for both early and late IFIs. Age, gender, underlying disease, and conditioning regimen were not significant.

Multivariate analyses showed previous history of IFI (hazard ratio $(\mathrm{HR})=9.53 ; 95 \%$ confidence interval (CI), 4.05-22.44), HLA mismatch ( $\mathrm{HR}=2.55$; 95\% CI, 1.07-6.06), sustained neutrophil $>2$ weeks $(\mathrm{HR}=1.94$; 95\% CI, 1.03-4.56), and Grades III to IV acute GVHD $(\mathrm{HR}=2.46 ; 95 \% \mathrm{CI}, 1.01-12.46)$ were significant for early IFI. A prior history of IFI $(\mathrm{HR}=2.62$; 95\% CI, 1.46-4.72), CMV disease ( $\mathrm{HR}=3.80 ; 95 \% \mathrm{CI}$, 1.71-8.42), limited and extensive chronic GVHD $(\mathrm{HR}=2.84 ; 95 \% \mathrm{CI}, 1.52-5.30$ and $\mathrm{HR}=3.21 ; 95 \% \mathrm{CI}$, 1.54-6.68, respectively) demonstrated a higher mortality risk for late IFI. The impact of corticosteroids for late IFI depended on the dosage. Corticosteroids of $<1.0 \mathrm{mg} /(\mathrm{kg} \cdot \mathrm{d})$ did not increase risk of developing IFI. Conversely, corticosteroids with a dosage of $\geq 1.0 \mathrm{mg} /(\mathrm{kg} \cdot \mathrm{d})$ affected IFI in the late period (Table 4).

\subsection{Outcomes}

IFI-related mortality was 49 out of 92 cases $(53.26 \%)$ of IFI. The 12-year overall survival (OS) rate for IFI was $41.9 \%$, with significant differences when compared with patients without IFI $(63.6 \%$, $P<0.001$; Fig. 3a). Late IFI was associated with a significantly higher 12-year OS compared with early IFI (Fig. 3b). For patients with only one IFI, the 12-year OS was better for patients with Candida when compared with molds (Fig. 3c).
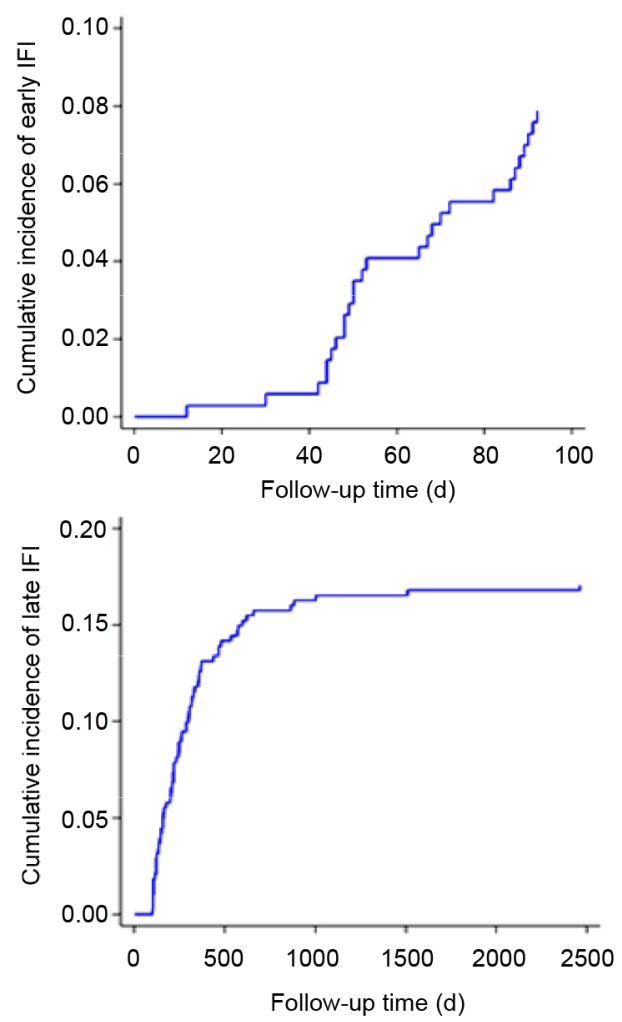

Fig. 1 Cumulative incidence rates of invasive fungal infection (IFI) among 408 patients after allo-HSCT The cumulative incidence rates of IFI for $100 \mathrm{~d}, 6$ months, and 1 year were $7.87 \%, 5.77 \%$, and $12.60 \%$, respectively

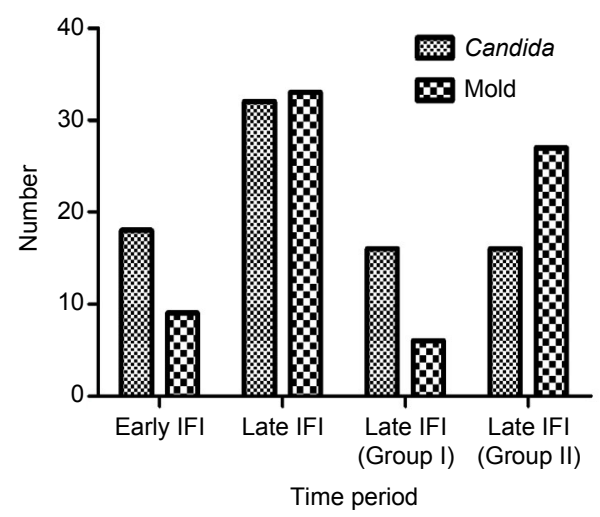

Fig. 2 Pathogen distribution of invasive fungal infection (IFI) in different time periods: early ( $\leq 100 \mathrm{~d}$ post-HSCT), late Group I (100-180 d post-HSCT), and late Group II ( $>180 \mathrm{~d}$ post-HSCT) 
Table 3 Univariate analysis of risk factors for early IFI ( $\leq 100 \mathrm{~d}$ post-HSCT) and late IFI ( $>100 \mathrm{~d}$ post-HSCT)

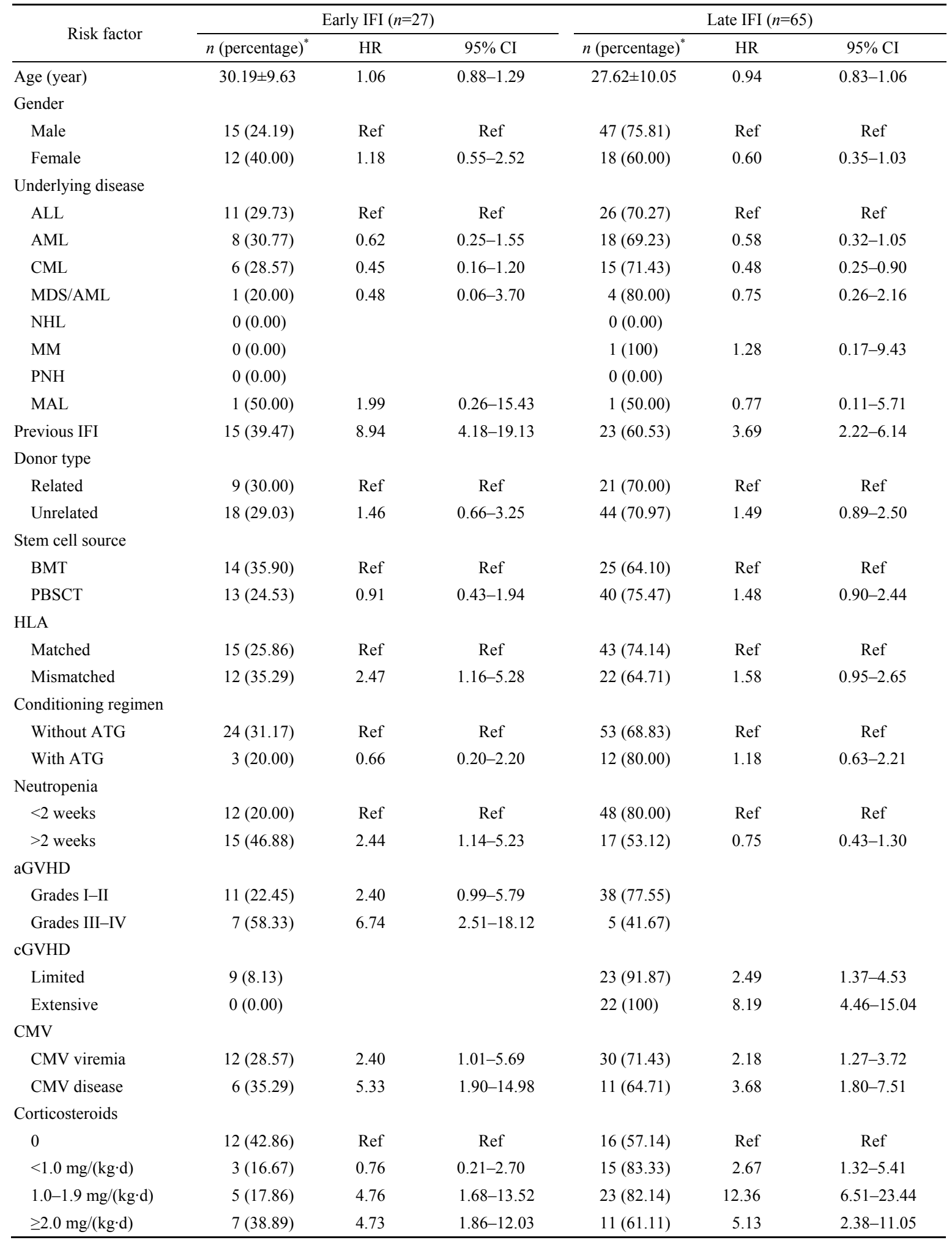

* Data are expressed as $n(\%)$, expect for age (mean \pm standard deviation). IFI: invasive fungal infection; ALL: acute lymphocytic leukemia; AML: acute myeloid leukemia; CML: chronic myeloid leukemia; MDS: myelodysplastic syndrome; NHL: non-Hodgkin's lymphoma; MM: multiple myeloma; PNH: paroxysmal nocturnal hemoglobinuria; MAL: mixed acute leukemia; BMT: bone marrow transplantation; PBSCT: peripheral blood stem cell transplantation; HLA: human leukocyte antigen; ATG: anti-thymocyte immunoglobuline; GVHD: graftversus-host-disease; aGVHD: acute GVHD; cGVHD: chronic GVHD; CMV: cytomegalovirus; Ref: reference 

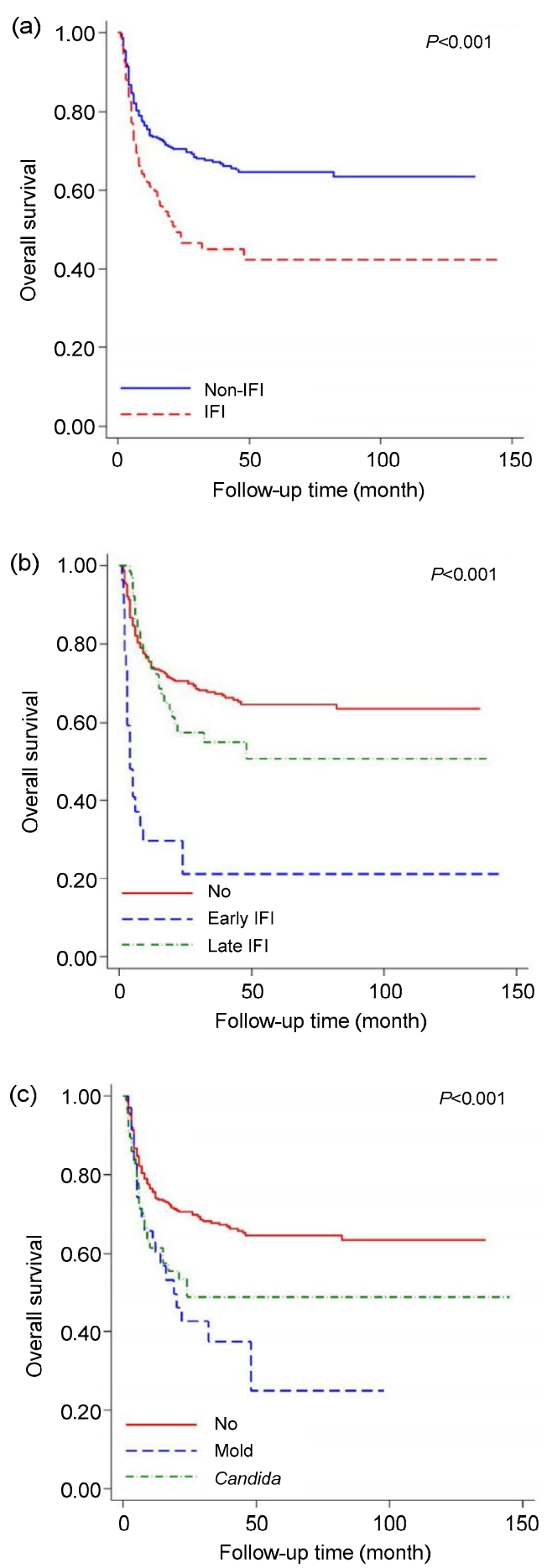

Fig. 3 Twelve-year overall survival of invasive fungal infection

(a) The overall survival for all 408 patients with or without IFI; (b) Overall survival for 27 patients with development of early IFI and 65 patients with late IFI; (c) Overall survival for patients with Candida infection and mold infection
Table 4 Multivariate analysis of risk factors for early and late IFIs

\begin{tabular}{|c|c|c|c|c|}
\hline \multirow{2}{*}{ Risk factor } & \multicolumn{2}{|c|}{ Early IFI $(n=27)$} & \multicolumn{2}{|c|}{ Late IFI $(n=65)$} \\
\hline & HR & $95 \% \mathrm{CI}$ & HR & $95 \% \mathrm{CI}$ \\
\hline Previous IFI & 9.53 & $4.05-22.44$ & 2.62 & $1.46-4.72$ \\
\hline \multicolumn{5}{|l|}{ HLA } \\
\hline Matched & Ref & Ref & & \\
\hline Mismatched & 2.55 & $1.07-6.06$ & & \\
\hline \multicolumn{5}{|l|}{ Neutropenia } \\
\hline$<2$ weeks & Ref & Ref & & \\
\hline$>2$ weeks & 1.94 & $1.03-4.56$ & & \\
\hline \multicolumn{5}{|l|}{ aGVHD } \\
\hline Grades I-II & 1.82 & $0.69-4.81$ & & \\
\hline Grades III-IV & 2.46 & $1.01-12.46$ & & \\
\hline \multicolumn{5}{|l|}{ cGVHD } \\
\hline Limited & & & 2.84 & $1.52-5.30$ \\
\hline Extensive & & & 3.21 & $1.54-6.68$ \\
\hline \multicolumn{5}{|l|}{ CMV } \\
\hline CMV viremia & 1.20 & $0.45-3.21$ & 1.13 & $0.63-2.03$ \\
\hline CMV disease & 2.88 & $0.92-8.95$ & 3.80 & $1.71-8.42$ \\
\hline \multicolumn{5}{|l|}{ Corticosteroids } \\
\hline 0 & Ref & Ref & Ref & Ref \\
\hline$<1.0 \mathrm{mg} /(\mathrm{kg} \cdot \mathrm{d})$ & 0.77 & $0.22-2.73$ & 1.80 & $0.87-3.73$ \\
\hline $1.0-1.9 \mathrm{mg} /(\mathrm{kg} \cdot \mathrm{d})$ & 2.06 & $0.58-7.27$ & 5.50 & $2.45-12.34$ \\
\hline$\geq 2.0 \mathrm{mg} /(\mathrm{kg} \cdot \mathrm{d})$ & 1.05 & $0.23-4.85$ & 2.74 & $1.14-6.64$ \\
\hline
\end{tabular}

IFI: invasive fungal infection; HLA: human leukocyte antigen; GVHD: graft-versus-host-disease; aGVHD: acute GVHD; cGVHD chronic GVHD; CMV: cytomegalovirus; Ref: reference

\section{Discussion}

Despite considerable progress in the management of the complications of HSCT, infection remains an important cause of post-transplant morbidity and mortality, primarily after allogeneic HSCT. European Group for Blood and Marrow Transplantation (EBMT) analyzed a large homogeneous group of 14403 patients transplanted for early leukemia from an HLA-identical sibling and cause of infection death after allogeneic HSCT (Gratwohl et al., 2005). Survival increased from $52 \%$ at 5 years in the first to $62 \%$ in the third cohort $(P<0.05)$, and treatment-related mortality decreased from $36 \%$ to $26 \%(P<0.05)$ due to a reduction in death from infection $(P<0.01)$. 
IFI is an important cause of post-transplant mortality. The incidence rate of IFI has been reported to be $10 \%-26 \%$ in HSCT recipients (Koldehoff and Zakrzewski, 2005; Post et al., 2007), with mortality ranging from $40 \%$ to $90 \%$ (Lin et al., 2001; Dagenais and Keller, 2009).

We performed this 12-year retrospective study to evaluate the epidemiologic characteristics, clinical manifestation and outcomes of IFI in 408 allogenic HSCT recipients. We report that patients with IFI have a lower 12-year OS than those without IFI ( $41.9 \%$ vs. $63.6 \%, P<0.01)$, reflecting the fact that IFI has a significant impact on long-term survival of transplant patients.

Over the past two decades, changes in transplantation practices and strategies to diagnose and treat IFI have likely impacted the epidemiology of IFI According to the data from the Transplant Associated Infections Surveillance Network, a network of 23 US transplant centers, invasive aspergillosis (43\%), invasive candidiasis $(28 \%)$, and zygomycosis $(8 \%)$ are the most common IFIs. Aspergillus fumigatus causes $44 \%$ of aspergillosis, and Candida glabrata is the most common organism $(33 \%)$ causing invasive candidiasis (Kontoyiannis et al., 2010). In our previous study, we performed CT-guided percutaneous lung biopsy in 16 patients who were initially suspected of developing fungal infection. Ten out of the 16 patients $(62.5 \%)$ were diagnosed with a fungal infection ( 8 with Aspergillus, 2 with mold fungus). All of the 10 cases had mold infection, and the incidence of Aspergillus infection was 80\% (Shi et al., 2009).

The observed results in our study are not entirely consistent with the prior studies. According to our data, candidiasis was more prevalent than mold infections (54.35\% vs. $45.65 \%)$. Among the candidiasis cases, Candida albicans was the most common pathogen (28\%), followed by Candida glabrata (18\%) and Candida tropicalis (14\%). The rate of mold infections was lower than that reported in our previous study (Shi et al., 2009), which may be explained by the following reasons. All patients underwent CT-guided lung biopsies in our previous prospective study, providing higher diagnostic accuracy than blood and sputum culture, while only 4 patients underwent biopsies in this retrospective study. Also, patients who received lung biopsies were highly suspected of Aspergillus infection based on imaging findings, probably leading to an increased rate of Aspergillus infection. Additionally, the small number of patients (16 cases) may also amplify the positive rate of mold infection.

Although Candida was presented as the most common pathogen in our center, there seemed to be a trend toward higher numbers of non-albicans and molds due to routine prophylaxis with fluconazole. This serves as a reminder to choose broad-spectrum antibiotics covering non-albicans and molds in the treatment of post-HSCT fungal infections. Since only a small number of patients were treated with voriconazole in our study, we observed no voriconazoleresistant species (e.g. Zygomycetes).

Previous studies have noted that IFI primarily occurs in the phase of hematopoietic reconstitution, generally two to three months after transplantation. Candida is the most common pathogen of early IFI, while mold is the most common pathogen of late IFI. In patients who also developed GVHD, IFI may occur at three to six months and even later (Koldehoff and Zakrzewski, 2005; Garcia-Vidal et al., 2008). Our data demonstrate that the median time for the development of ICI is earlier than that for IMI (140 d vs. $243 \mathrm{~d}, P<0.05)$, reflecting the fact that mold infections are more common in the late period after HSCT. The incidences of mold and Candida infections in early and late IFIs differed, but did not reach statistical significance. We further evaluated the incidence rate of very late IFI and found that mold infections significantly increased $(P<0.05)$. These findings highlight the importance of strengthening the detection and treatment of mold in the late period after transplantation.

Multiple factors reported to be associated with the risk of IFI include donor type, conditioning regimens, prolonged neutropenia, GVHD, and highdose corticosteroids (Fukuda et al., 2003; Thursky et al., 2004; Ozyilmaz et al., 2010; Omer et al., 2013). According to our data, we found similar risk factors infecting IFI. However, when we further analyzed the risk factors for each phase of IFI, we were able to demonstrate that there is a difference between the early and late IFIs.

Despite the widespread use of cyclosporine, tacrolimus, mycophenolate mofetil, and other drugs for prophylaxis of GVHD in HSCT recipients, $25 \%$ to $45 \%$ of patients still developed Grades II to IV acute GVHD. HLA-mismatched recipients are at a particularly 
high risk for acute GVHD, which is the most likely cause of the high risk for IFI, especially for early IFI. Enhancing the immune inhibitors or increasing the dosage of corticosteroids may effectively control GVHD, while increasing the risk of IFI.

In our study, univariate analysis showed that corticosteroid therapy is a risk factor during all periods. When further introduced into multivariate analyses, corticosteroid therapy only accounted for late IFI. This result suggests that long-term use of corticosteroids has a more advanced effect on late IFI.

CMV is associated with development of IFI in both the early and the late post-transplant phases (Marr et al., 2002). The mechanism remains unclear. The immune-modulating effect of CMV may be one of the explanations. In our study, CMV infection was the risk factor for early IFI in univariate analysis but not in multivariate analyses. CMV disease rather than CMV infection was associated with the risk of late IFI. This may be because CMV mostly occurs in the late period after transplantation. Strengthening serum monitoring of CMV and timely prevention of CMV may reduce the incidence of IFI, particularly late IFI.

Prolonged neutropenia is a risk factor for IFI (Hovi et al., 2000). Neutrophils play an important role in the defense against IFI. Our results showed that prolonged neutropenia, which generally occurs in the early period after transplantation, is a risk factor for early IFI but not for late IFI.

It is important to recognize that the risks for IFI also depend on changes in the conditioning regimens, supportive care strategies, and therapy strategies. This study attempts to analyze the impact of conditioning regimens of IFI, such as myeloablative and nonmyeloablative regimens, as well as conditioning regimens with or without ATG. Unfortunately we cannot draw convincing conclusions due to the small number of cases. Further work will be needed to assess the association between conditioning regimen and IFI.

It should be noted that our study has some limitations. Firstly, incidence of IFI can be lower than the actual incidence because of the strict definition of IFI (our study did not include possible IFI cases). Secondly, some patients with IFI may be misplaced into the control group, thus the significance of the difference between the two groups may be diluted. What is more, 125 of the total 408 patients participated in clinical trials of secondary prophylaxis during the study period, and patients with a history of IFI pre-HSCT received itraconazole $(200 \mathrm{mg} / \mathrm{d}$, i.v. $)$ during neutropenia $\left(<1.0 \times 10^{9} \mathrm{~L}^{-1}\right)$, and changed to oral fluconazole or itraconazole after neutrophil recovery $\left(>1.0 \times 10^{9} \mathrm{~L}^{-1}\right)$, which in part may reduce the incidence of IFI after HSCT.

\section{Acknowledgements}

We thank the HLA Typing Laboratory of the Blood Center of Zhejiang Province (China) for their assistance.

\section{Compliance with ethics guidelines}

Ji-min SHI, Xu-ying PEI, Yi LUO, Ya-min TAN, Ru-xiu TIE, Jing-song HE, Wei-yan ZHENG, Jie ZHANG, Zhen CAI, Mao-fang LIN, and He HUANG declare that they have no conflict of interest.

All procedures followed were in accordance with the ethical standards of the responsible committee on human experimentation (institutional and national) and with the Helsinki Declaration of 1975, as revised in 2008 (5). Informed consent was obtained from all patients for being included in the study. Additional informed consent was obtained from all patients for which identifying information is included in this article.

\section{References}

Ascioglu, S., Rex, J.H., de Pauw, B., et al., 2002. Defining opportunistic invasive fungal infections in immunocompromised patients with cancer and hematopoietic stem cell transplants: an international consensus. Clin. Infect. Dis., 34(1):7-14. [doi:10.1086/323335]

Bjorklund, A., Aschan, J., Labopin, M., et al., 2007. Risk factors for fatal infectious complications developing late after allogeneic stem cell transplantation. Bone Marrow Transplant., 40(11):1055-1062. [doi:10.1038/sj.bmt.170 5856]

Dagenais, T.R., Keller, N.P., 2009. Pathogenesis of Aspergillus fumigatus in invasive aspergillosis. Clin. Microbiol. Rev., 22(3):447-465. [doi:10.1128/CMR.00055-08]

Fukuda, T., Boeckh, M., Carter, R.A., et al., 2003. Risks and outcomes of invasive fungal infections in recipients of allogeneic hematopoietic stem cell transplants after nonmyeloablative conditioning. Blood, 102(3):827-833. [doi:10.1182/blood-2003-02-0456]

Garcia-Vidal, C., Upton, A., Kirby, K.A., et al., 2008. Epidemiology of invasive mold infections in allogeneic stem cell transplant recipients: biological risk factors for infection according to time after transplantation. Clin. Infect. Dis., 47(8):1041-1050. [doi:10.1086/591969]

Gratwohl, A., Brand, R., Frassoni, F., et al., 2005. Cause of death after allogeneic haematopoietic stem cell transplantation (HSCT) in early leukaemias: an EBMT analysis of lethal infectious complications and changes over calendar time. Bone Marrow Transplant., 36(9):757-769. [doi:10.1038/sj.bmt.1705140] 
Hovi, L., Saarinen-Pihkala, U.M., Vettenranta, K., et al., 2000. Invasive fungal infection in pediatric BMT recipients: single center experience of 10 years. Bone Marrow Transplant., 26(9):999-1004. [doi:10.1038/sj.bmt.1702654]

Jantunen, E., Ruutu, P., Niskanen, L., et al., 1997. Incidence and risk factors for invasive fungal infections in allogeneic BMT recipients. Bone Marrow Transplant., 19(8): 801-808. [doi:10.1038/sj.bmt.1700737]

Koldehoff, M., Zakrzewski, J.L., 2005. Modern management of respiratory failure due to pulmonary mycoses following allogeneic hematopoietic stem-cell transplantation. Am. J. Hematol., 79(2):158-163. [doi:10.1002/ajh.20361]

Kontoyiannis, D.P., Marr, K.A., Park, B.J., et al., 2010. Prospective surveillance for invasive fungal infections in hematopoietic stem cell transplant recipients, 2001-2006: overview of the Transplant Associated Infection Surveillance Network (TRANSNET) Database. Clin. Infect. Dis., 50(8):1091-1100. [doi:10.1086/651263]

Lin, S.J., Schranz, J., Teutsch, S.M., 2001. Aspergillosis casefatality rate: systematic review of the literature. Clin. Infect. Dis., 32(3):358-366. [doi:10.1086/318483]

Marr, K.A., Carter, R.A., Boeckh, M., et al., 2002. Invasive aspergillosis in allogeneic stem cell transplant recipients: changes in epidemiology and risk factors. Blood, 100(13): 4358-4366. [doi:10.1182/blood-2002-05-1496]

Neofytos, D., Horn, D., Anaissie, E., et al., 2009. Epidemiology and outcome of invasive fungal infection in adult hematopoietic stem cell transplant recipients: analysis of Multicenter Prospective Antifungal Therapy (PATH) Allianee registry. Clin. Infect. Dis., 48(3):265-273. [doi:10. 1086/595846]

Omer, A.K., Ziakas, P.D., Anagnostou, T., et al., 2013. Risk factors for invasive fungal disease after allogeneic hematopoietic stem cell transplantation: a single center experience. Biol. Blood Marrow Transplant., 19(8):1190-1196. [doi:10.1016/j.bbmt.2013.05.018]

Ozyilmaz, E., Aydogdu, M., Sucak, G., et al., 2010. Risk factors for fungal pulmonary infections in hematopoietic stem cell transplantation recipients: the role of iron overload. Bone Marrow Transplant., 45(10):1528-1533. [doi:10.1038/bmt.2009.383]

Post, M.J., Lass-Floerl, C., Gastl, G., et al., 2007. Invasive fungal infections in allogeneic and autologous stem cell transplant recipients: a single-center study of 166 transplanted patients. Transplant Infect. Dis., 9(3):189-195. [doi:10.1111/j.1399-3062.2007.00219.x]
Shi, J.M., Cai, Z., Huang, H., et al., 2009. Role of CT-guided percutaneous lung biopsy in diagnosis of pulmonary fungal infection in patients with hematologic diseases. Int. J. Hematol., 89(5):624-627. [doi:10.1007/s12185-0090351-0]

Thursky, K., Byrnes, G., Grigg, A., et al., 2004. Risk factors for post-engraftment invasive aspergillosis in allogeneic stem cell transplantation. Bone Marrow Transplant., 34(2): 115-121. [doi:10.1038/sj.bmt.1704543]

\section{中文概要}

题 目: 异基因造血干细胞移植术后侵袭性真菌感染的流 行病学: 单中心 12 年研究结果

目 的: 回顾性分析了浙江大学医学院附属第一医院骨髓 移植中心 1998 年至 2009 年接受造血干细胞移植

(HSCT) 发生侵袭性真菌感染（IFI）的患者资 料, 评估 HSCT 后 IFI 发病情况、流行病学特征 及危险因素, 以期寻找病原菌特点及相关的危险 因素, 为制定更有针对性的预防治疗策略提供依 据。

创新点: 首次对本中心异基因 HSCT 后的 IFI 感染情况进 行统计分析, 研究年限长, 样本量较大; 另作为 国内较成熟的移植中心之一, 本中心的研究结果 对国内异基因 HSCT 后 IFI 感染情况有较大的参 考意义。

方 法: 对 1998 年 11 月至 2009 年 12 月在本移植中心接 受异基因 HSCT 的 408 例患者进行观察分析, 总 结 HSCT 后 IFI 发病情况和病原菌分布, 并采用 多因素分析判断危险因素。

结 论: 本研究显示 IFI 与 HSCT 预后明显相关, HSCT 后不同时期 IFI病原菌分布及危险因素存在差异。 临床应根据 IFI 病原学特点及 HSCT 后时间分布 特点, 采取不同的策略, 更有针对性地用药及开 始治疗, 从而改善预后。

关键词: 侵袭性真菌感染; 异基因造血干细胞移植; 危险 因素 